\title{
Primary lung cancer firstly presents as nephrotic syndrome: one case report and literature review
}

\author{
Dongmei Gu ${ }^{1 \#}$, Wenjie Liao ${ }^{2 \#}$, Qingqing $\mathrm{Su}^{3}, \mathrm{Jie} \mathrm{Gu}^{3}$, Yanbin $\mathrm{Chen}^{3}$ \\ ${ }^{1}$ Department of Pathology, the First Affiliated Hospital of Soochow University, Suzhou 215000, China; ${ }^{2}$ Emergency Medical Center, The Second \\ People's Hospital of Lianyungang, Lianyungang 222000, China; ${ }^{3}$ Department of pulmonary and critical care medicine, the First Affiliated Hospital \\ of Soochow University, Suzhou 215000, China \\ "These authors contributed equally to this work. \\ Correspondence to: Prof. Yanbin Chen. Department of Pulmonary and Critical Care Medicine, The First Affiliated Hospital of Soochow University, \\ 899\#, Pinghai Road, Suzhou 215000, China. Email: chen001chen@163.com.
}

\begin{abstract}
Primary lung cancer presents as nephrotic syndrome firstly is a rare condition. Few data are available to address this question because of its seldom. Herein we present one case of primary lung cancer which showing nephrotic syndrome as first manifestation and review the literature.
\end{abstract}

Keywords: Nephrotic syndrome; membranous nephropathy (MN); paraneoplastic; lung cancer; case report

Submitted Oct 06, 2019. Accepted for publication Nov 05, 2019.

doi: $10.21037 /$ tcr.2019.11.18

View this article at: http://dx.doi.org/10.21037/tcr.2019.11.18

\section{Introduction}

Nephrotic syndrome is defined as a constellation of clinical signs and symptoms including massive proteinuria (urine protein $\geq 3.5 \mathrm{~g} / \mathrm{d}$ ), hypoalbuminemia (serum albumin $\leq 3 \mathrm{~g} / \mathrm{dL}$ ), peripheral edema and hyperlipidemia. Nephrotic syndrome is a rare condition, the annual incidence in adults is three per 100,000 persons. Approximately $80 \%$ to $90 \%$ of nephrotic syndrome cases in adults are idiopathic (1). Nephrotic syndrome has been considered as a potential paraneoplastic process for approximately one century which may precede the onset of the primary tumor, and the eradication of initial tumor can result in the remission of nephrotic syndrome (2). There is enough literature data regarding the association between nephrotic syndrome and different neoplasia, but only few cases address the specific link between nephrotic syndrome and lung cancer, especially when nephrotic syndrome firstly presents as paraneoplastic syndrome of primary lung cancer (3). Herein, we present one case of primary lung cancer which showing nephrotic syndrome as first manifestation and retrospectively evaluated the characteristics of nephrotic syndrome as first manifestation of primary lung cancer.

\section{Case presentation}

A male patient aged 65 years old who was receiving treatment for membranous nephropathy $(\mathrm{MN})$ for ten months was referred and hospitalized in our hospital because of cough and expectoration. He had a history of bubble urine for one year before hospitalization. Ten months ago, he suffered from fatigue and weight loss, so he consulted at other hospital and underwent urinalysis which revealed proteinuria $3(+)$ with urinary occult blood positive and normal renal function, his level of proteinuria on successive check-ups was over $3.5 \mathrm{~g} /$ day. His serum albumin level decreased to $2.4 \mathrm{~g} / \mathrm{dL}$ (normal range, $4.0-5.5 \mathrm{~g} / \mathrm{dL}$ ). His chest computed tomography (CT) scan was negative. Subsequently, renal biopsy was performed and histopathological findings was characteristic for $\mathrm{MN}$. During his ten-month therapy for $\mathrm{MN}$, his urinalysis and serum chemistry data showed that the proteinuria, hypoalbuminemia and hypercholesterolemia did not improve. He was a smoker of 20 cigarettes/day for 15 years. He did not drink alcohol, or use intravenous drugs. His family history, psycho-social history and genetic information were unremarkable. On examination, his respiratory frequency was 18 breaths/min, heart rate was 90 beats/min, blood pressure was 106/62 mmHg, body temperature was $36.9^{\circ} \mathrm{C}$ and his oxygen saturation was $100 \%$ on room air. Chest examination revealed nothing remarkable and edema of the lower limbs was notable. 

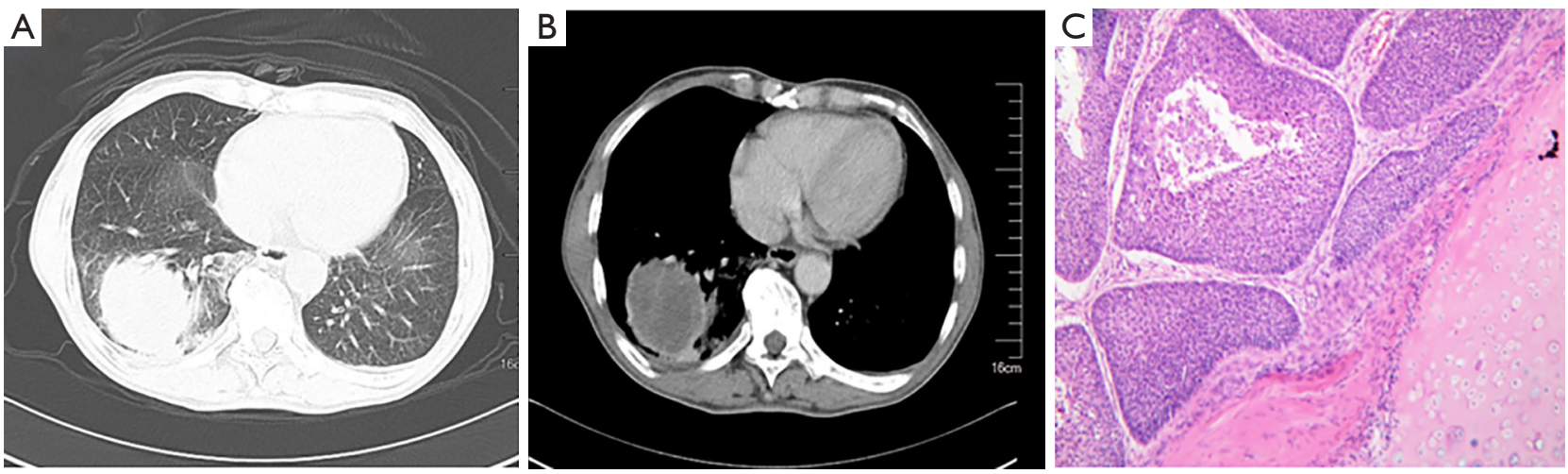

Figure 1 Radiological changes and pathological features of the patient. (A) Chest computed tomography scan (lung window) shows a mass located in the right lower lobe measured nearly $5.0 \mathrm{~cm}$ in diameter; (B) chest computed tomography scan (mediastinal window) shows the mass measured in same size; (C) squamous carcinoma cells are shown on hematoxylin-eosin staining (40x).

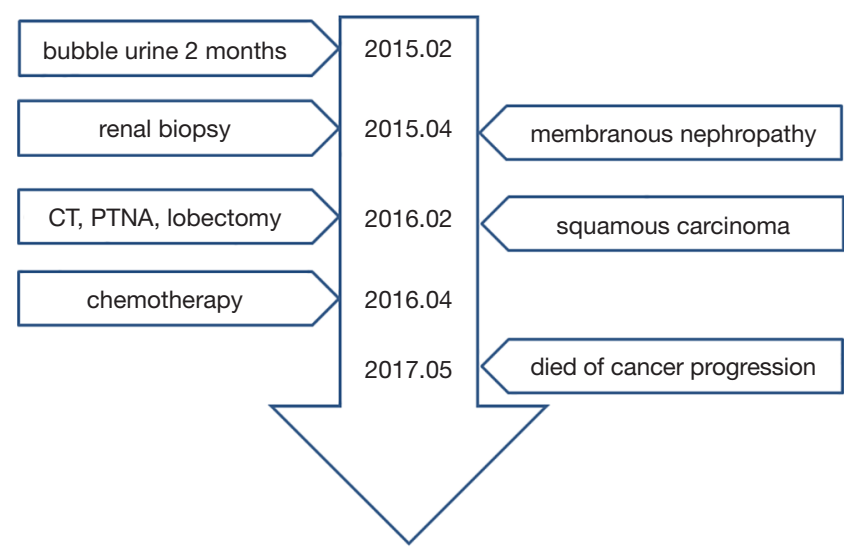

Figure 2 The timeline picture of the patient. CT, computed tomography; PTNA, percutaneous needle aspiration.

On admission, we arranged chest CT scan for him, which revealed a tumor of $5 \mathrm{~cm}$ in diameter at right lower lobe without enlarged lymph nodes (Figure 1A,B). CT-guided percutaneous needle aspiration (PTNA) was performed and histopathology revealed squamous carcinoma (Figure 1C). The patient underwent right lower lobectomy, which revealed a neoplastic mass with a diameter of $5 \mathrm{~cm}$. Histopathology confirmed the diagnosis and the tumor was classified as T3N2M0 (stage IIIA). Postoperatively, the patient received docetaxel $120 \mathrm{mg}$ day 1 plus nedaplatin 100 $\mathrm{mg}$ day 1 chemotherapy for four cycles. And his proteinuria disappeared, his serum albumin level increased to $5.5 \mathrm{~g} / \mathrm{dL}$. His membranous nephropathy got completely remission. But he denied further therapy on lung cancer, and fifteen months after surgery, the patient died because of relapse of cancer and accompanied deterioration of MN. The timeline picture of the patient was shown in Figure 2.

\section{Discussion}

It is well known that $\mathrm{MN}$ is the most common nephropathy associated with neoplasm. The vast majority of tumors associated with $\mathrm{MN}$ are lung cancer and prostate cancer. Primary lung cancer is responsible for about $3 \%$ of all cases of membranous nephropathy. The highest prevalence is observed in patients older than 60 years.

The interval between nephrotic syndrome and the malignancy is variable. Nephrotic syndrome occurs before the diagnosis of cancer in approximately $40 \%$ of patients, at the time of diagnosis in $40 \%$ of patients, and after diagnosis in $20 \%$ of patients (4). Median time from diagnosis of nephrotic syndrome to diagnosis of cancer is 60 months, which suggests that the predilection for cancer in participants with nephrotic syndrome persists for many years. As to our case, he was diagnosed as $\mathrm{MN}$ firstly, but his condition did not improve after he received treatment on $\mathrm{MN}$. The 10-month therapy result alerted us that the MN maybe secondary to some kind of unrecognized disease. So after admission, we arranged CT scan for him and eventually confirmed that lung cancer was the reason of his nephrotic syndrome. The rare case we present will contribute to understanding the clinical characteristics of $\mathrm{MN}$ and lung cancer. Among all types of lung cancer, squamous cancer and adenocarcinoma are the most common pathologic type which presents nephrotic syndrome as first manifestation.

The exactly mechanism whereby cancer might be associated with nephrotic syndrome has yet to be elucidated. The most accepted potential mechanisms are listed as 
follows: firstly, antibodies induced by tumor antigen immunologically similar to an endogenous podocyte antigen, which can cause immune complex formation in situ; secondly, circulating antibodies could interact with tumor antigens planted in subepithelial location; thirdly, shedding tumor antigens form circulating immune complexes, then trap in the glomerular capillary wall. C-Maf-inducing protein and thrombospondin type- 1 domain containing $7 \mathrm{~A}$ may also play some roles in the development of paraneoplastic nephrotic syndrome (5).

The curative treatment of the primary tumor is effective in the cure of nephrotic syndrome. Nephrotic syndrome can completely or partially resolve when lung cancer treated curatively with chemotherapy, radiotherapy or surgery. One study showed that successful treatment of primary lung cancer leads to improvement or disappearance of proteinuria in $75 \%$ and complete regression of nephrotic syndrome could be expected in $25 \%$ (6). The 1 -year mortality in patients of cancer with preceding nephrotic syndrome was $40.8 \%$, the 5 -year mortality was $68.5 \%$.

There are some limitations in our report. Firstly, the patient was diagnosed as $\mathrm{MN}$ in the previous hospital and received appropriate therapy on $\mathrm{MN}$, but his condition did not improve. The 10 -month period was a diagnostic challenge, of course, it still was a great chance for making definite diagnosis. If the clinicians in that hospital arranged chest CT scan for him, the result may be quite different. Secondly, after the patient received four cycles of chemotherapy on lung cancer and got completely remission of $\mathrm{MN}$, he denied further therapy. If he continued follow the doctors' advice, his overall survival time maybe even longer.

\section{Conclusions}

Primary lung cancer presents as nephrotic syndrome firstly is a rare condition, Clinicians must pay close attention to the patients of nephrotic syndrome from the moment of diagnosis and throughout the whole follow-up period in order to detect lung cancer as early as possible.

\section{Acknowledgments}

Funding: None.

\section{Footnote}

Conflicts of Interest: All authors have completed the ICMJE uniform disclosure form (available at http://dx.doi. org/10.21037/tcr.2019.11.18). The authors have no conflicts of interest to declare.

Ethical Statement: The authors are accountable for all aspects of the work in ensuring that questions related to the accuracy or integrity of any part of the work are appropriately investigated and resolved. All procedures performed in studies involving human participants were in accordance with the ethical standards of the institutional and/or national research committee(s) and with the Helsinki Declaration (as revised in 2013). Consent was obtained from relative of the patient for publication of this report and any accompanying images.

Open Access Statement: This is an Open Access article distributed in accordance with the Creative Commons Attribution-NonCommercial-NoDerivs 4.0 International License (CC BY-NC-ND 4.0), which permits the noncommercial replication and distribution of the article with the strict proviso that no changes or edits are made and the original work is properly cited (including links to both the formal publication through the relevant DOI and the license). See: https://creativecommons.org/licenses/by-nc-nd/4.0/.

\section{References}

1. Christiansen CF, Onega T, Sværke C, et al. Risk and prognosis of cancer in patients with nephrotic syndrome. Am J Med 2014;127:871-7.e1.

2. Tadokoro A, Ishii T, Takahama T, et al. Paraneoplastic focal segmental glomerulosclerosis in a patient with lung adenocarcinoma. Intern Med 2013;52:1953-6.

3. Bacchetta J, Juillard L, Cochat P, et al. Paraneoplastic glomerular diseases and malignancies. Crit Rev Oncol Hematol 2009;70:39-58.

4. Ohara G, Satoh H, Kurishima K, et al. Paraneoplastic nephrotic syndrome in patients with lung cancer. Intern Med 2009;48:1817-20.

5. Hoxha E, Wiech T, Stahl PR, et al. A Mechanism for Cancer-Associated Membranous Nephropathy. N Engl J Med 2016;374:1995-6.

6. Malinowska M, Doubel P, Gheysens B, et al. Nephrotic syndrome as first manifestation of a bronchopulmonary malignancy. A case report. Acta Chir Belg 2010;110:350-3.

Cite this article as: Gu D, Liao W, Su Q, Gu J, Chen Y. Primary lung cancer firstly presents as nephrotic syndrome: one case report and literature review. Transl Cancer Res 2019;8(8):2933-2935. doi: 10.21037/tcr.2019.11.18 